\title{
Evidence for a shift in the choice criterion of rats in a 12-arm radial maze
}

\author{
MICHAEL F. BROWN \\ Villanova University, Villanova, Pennsylvania \\ and \\ EVANGELINE A. WHEELER and DONALD A. RILEY \\ University of California, Berkeley, California
}

\begin{abstract}
Rats were trained in a standard 12 -arm radial maze task. Following training, each trial consisted of a sequence of $2,4,6,8$, or 10 choices, followed by a 15-min delay, which then was followed by a choice between a single arm and a response manipulandum mounted in the center of the maze. An arm visit was reinforced if the arm had not been visited prior to the delay, whereas a manipulandum response was reinforced if the arm had been visited. It was found that rats are relatively more likely to reject arms by responding to the manipulandum following a delay occurring late in the choice sequence. This indicates that the choice criterion used by rats in the radial maze becomes more strict as the choice sequence progresses. Such a process provides an alternative explanation for some of the data recently reported by Cook, Brown, and Riley (1985).
\end{abstract}

The past decade has witnessed a great deal of research aimed at understanding the memory processes used by rats while solving spatial tasks. This research has been stimulated in large part by Olton's introduction of the radial maze paradigm (e.g., Olton, 1978; Olton \& Samuelson, 1976). One recent focus of this research has been the manner in which the rat's psychological processes change over the course of the choice sequence that constitutes each trial in the radial maze. On each trial, the rat makes a series of choices from among the maze arms until the food on each arm has been eaten. There are a number of factors that may vary over the course of each trial: the animal may become sated as the choice sequence progresses, the amount of information encoded in memory may change, retroactive and/or proactive interference may occur, and so forth. The logical properties of the task also change as a function of choice number. For example, the proportion of arms that remain baited decreases as a function of choice number. These dynamic properties of the radial maze task and their effects on psychological processes have only recently come under experimental study (e.g., Brown \& Cook, 1986; Cook, Brown, \& Riley, 1985).

Cook et al. (1985) presented evidence that the type of representation present in working memory during a delay interpolated in a 12-arm radial maze task depends on whether the delay occurs early or late in the choice sequence. Specifically, they argued that rats retain represen-

This research was supported by National Science Foundation Grant BNS-8317577 to D. A. Riley. Support during preparation of the manuscript was provided by National Institute of Mental Health Grant RO1 MH42646 to M. F. Brown. Correspondence concerning this article should be addressed to Michael F. Brown, Department of Psychology, Villanova University, Villanova, PA 19085. tations of the previously visited arms of the maze if the delay occurs early in the choice sequence (retrospective memory), but retain representations of anticipated to-bevisited arms if the delay occurs late in the choice sequence (prospective memory). Prospective memory has been used to explain a number of findings in the literature on conditional discriminations in pigeons (for reviews, see Honig \& Thompson, 1982; Riley, Cook, \& Lamb, 1981; Wasserman, 1986). Such anticipatory representation of future choices is logically possible in the radial-arm maze because the rat is presented with the same set of items (arms of the maze) for large numbers of sessions. The nominal task of avoiding revisits requires discrimination of previously visited arms from unvisited arms. If longterm information about the characteristics of the maze is available in reference memory (Honig, 1978) and/or in a "cognitive map" (O'Keefe \& Nadel, 1978), then it would be possible to determine which arms remain to be visited given information about which arms have been visited.

Cook et al. (1985) inserted a delay after the 2nd, 4th, 6th, 8th, or 10th choice in a 12-arm radial maze (this experimental variable is referred to subsequently as point of interpolation, or POI). Two results led to the conclusion that the content of working memory is retrospective during delays with an early POI, but prospective during delays with a late POI. First, a 15-min delay inserted in the middle of the choice sequence (i.e., after the 6th choice) disrupted choice accuracy more than did the same delay inserted either earlier in the choice sequence or later in the choice sequence. Assuming that the amount of disruption produced by the delay reflects the amount of information retained during the delay, this pattern of results implies that less information was being retained when the 
delay occurred early or late in the sequence than when the delay occurred in the middle of the sequence. Therefore, rats must remember the few visited arms during an early delay, but the few to-be-visited arms during a late delay. Thus, Cook et al. concluded that rats use either retrospective or prospective memory, depending on which representation contains the least amount of information.

Second, evidence for this dual-coding hypothesis was provided by an analysis of the source of errors made following the delay. In the early POI conditions, revisits to earlier chosen arms were more likely than revisits to arms chosen more recently. This finding is an example of a recency effect; more recently experienced items (maze arms) were better remembered. A recency effect would be expected if rats avoided revisits by retaining retrospective representations of visited arms and if memory is poorer for more remote events, because the order in which arms were visited would map onto the order in which they were encoded in memory. In late POI conditions, Cook et al. (1985) found no evidence of a recency effect. None would be expected if rats were not retaining representations of visited arms, but were instead retaining representations of anticipated choices. This is because the contents of working memory would consist of those items not yet presented, a set that is irrelevant to the ordinal positions of the presented items (see Cook et al., 1985, p. 455).

Although Cook et al. (1985) concluded that the quadratic function relating POI and the effect of a 15-min delay supports the dual-coding hypothesis, they acknowledged that an alternative explanation was possible. This criterion-shift hypothesis proposes that two independent processes interact to produce the pattern of results. First, retrospective memory is used during the delay, regardless of POI. Thus, the later the POI, the larger the memory load during the delay. This explains the fact that a delay interpolated in the middle of the choice sequence is more disruptive than a delay interpolated early in the choice sequence. In addition, rats adopt an increasingly strict criterion for visiting arms as the choice sequence progresses. This criterion shift may be produced by the decrease in the proportion of arms that are baited as the choice sequence progresses. Following an early delay, most arms remain baited. Thus, a randomly chosen arm is likely to contain food. Following a late delay, on the other hand, most arms have been depleted and a randomly chosen arm is unlikely to be baited. In terms of signal detection theory (Green \& Swets, 1966), a choice criterion might be adjusted based on the baseline probability of reinforcement. When most arms remain baited (early in the choice sequence), the choice criterion is relatively lax. As the proportion of arms baited decreases (late in the choice sequence), the choice criterion becomes more strict.

Operationally, the radial maze task consists of a series of choices in which one arm is chosen from among the entire set. However, it seems quite reasonable to suppose that each of these observable macrochoices consists of a series of sequential microchoices, in which individual arms are evaluated and either accepted or rejected. If accepted, an observable macrochoice is exhibited as the rat visits the chosen arm. Rejection of an arm, on the other hand, results in another microchoice. Presumably, a number of microchoices might result in rejections before an arm is finally accepted at the microchoice level, resulting in a macrochoice. This view of rat choice behavior in mazes has an extensive history (e.g., Bower, 1959; Still, 1976) and has been encouraged by studies of vicarious trial-and-error behavior (Muenzinger, 1938; Tolman, 1938).

The criterion-shift hypothesis can be formalized in the context of this distinction between macrochoices and microchoices in the radial maze. The hypothesis claims that macrochoice accuracy is determined by two independent processes. First, retrospective coding of previous choices results in increasingly difficult discriminations at the microchoice level as the delay is interpolated later in the choice sequence. Second, a stricter criterion for choosing arms results in a higher rate of rejecting arms at the microchoice level, thereby increasing accuracy at the macrochoice level by increasing the number of arms that are evaluated before a macrochoice occurs.

Figure 1 illustrates the criterion-shift theory in a form that was inspired by Wright and Sand's (1981) model of pigeon matching-to-sample behavior. It depicts hypothetical distributions of psychological effects produced during microchoices in the radial maze. The exact nature of these effects is unknown and therefore unspecified. However, it may be useful as a heuristic to consider values on the scale to correspond to the "familiarity" of arms evaluated during the microchoice, with values toward the left being more familiar (remembered) and values toward the right being less familiar (not remembered). In each of the three panels, the $O$ distribution represents effects produced by previously visited (old) arms. The $N$ distribution represents effects produced by unvisited (new) arms. All arms that produce effects falling to the left of the dotted criterion line are classified as previously visited $(o)$, and hence are not visited. Arms producing effects falling to the right of the criterion are classified as not previously visited (n), and hence are visited. Thus, in signal detection terms, the $N_{n}, O_{0}, N_{o}$, and $O_{n}$ areas represent microchoice outcomes of hit (correctly visiting a previously unvisited arm), correct rejection (correctly rejecting a previously visited arm), miss (incorrectly rejecting a previously unvisited arm), and false alarm (incorrectly visiting a previously visited arm), respectively. It should be noted that although this illustration of the criterionshift theory assumes that microchoices produce distributions of effects that are approximately normal, this assumption will not be necessary for the formal models presented in the Discussion.

Each panel of Figure 1 describes the microchoice outcomes in a different POI condition as predicted by the criterion-shift theory. The decrease in distance between the means of the $N$ and $O$ distributions as a function of POI reflects the increasing difficulty of the discrimina- 


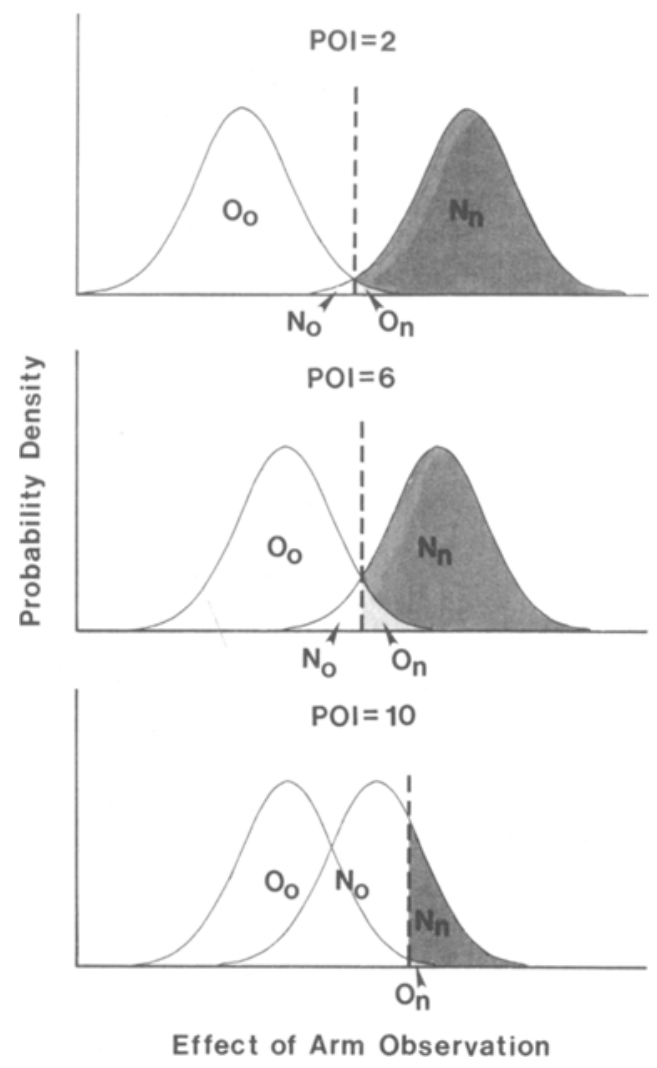

Figure 1. An illustration of the criterion-shift hypothesis. The $O$ distribution represents the effects produced by old (visited) arms, and the $\boldsymbol{N}$ distribution represents the effects produced by new (unvisited) arms. During each microchoice, arms are classified as either old (left side of the dotted criterion line) or new (right side of the criterion), which results in either a rejection or a macrochoice, respectively. According to the hypothesis, $d^{\prime}$ decreases as a function of point of delay interpolation (POI), while the criterion shifts in a manner that increases the probability of a rejection (see text for further explanation).

tion produced, at least in part, by an increase in retrospective memory load. The difference in the position of the criterion line reflects the proposed criterion shift. In particular, an arm must be less familiar following a delay interpolated late in the choice sequence to produce a macrochoice than is required if the delay had occurred earlier in the choice sequence.

In terms of this illustration, accuracy at the macrochoice level is given by

$$
N_{n} /\left(N_{n}+O_{n}\right)
$$

that is, observable macrochoice accuracy is given by correct visits divided by total visits. It is evident from an inspection of the figure that a combination of decreasing distance between $N$ and $O$ and a shift in the choice criterion could produce a quadratic function relating POI and macrochoice accuracy.

The present experiment was an attempt to test the criterion-shift hypothesis. A procedure was used in which, following a delay interpolated in the choice sequence, the rat was exposed to a single arm and either accepted (visited) or rejected (did not visit) that arm. Thus, the basic strategy was to isolate microchoices and measure their outcome. During each trial, rats first visited 2, 4, 6, 8, or $10 \mathrm{arms}$ in a 12-arm radial maze. The sequence of arms was randomly determined to rule out any influence of response biases or algorithms (Olton, 1978). Following a 15-min retention interval, the rats were given a choice between visiting a single arm and pressing a manipulandum located in the central arena of the maze. If the arm had been visited prior to the delay, then it was unbaited so that a visit to it was not reinforced. On these trials, a response to the manipulandum produced food reinforcement. If, on the other hand, the arm had not been visited prior to the delay, then the arm was baited, but a response to the manipulandum had no effect.

This experimental design allows the use of signal detection indices (Green \& Swets, 1966; Swets, Tanner, \& Birdsall, 1961) to test the predictions of the criterion-shift hypothesis. The task of choosing between the arm and the center manipulandum following the delay is functionally equivalent to a go/no-go signal detection task. When the arm has not been visited prior to the delay (and therefore remains baited), an arm visit is considered to be a hit, and a manipulandum response is a miss. Likewise, if the arm has been visited prior to the delay (and is therefore unbaited), an arm visit is a false alarm and a manipulandum response is a correct rejection. From the relative proportions of these response classes, the two major parameters of signal detection theory can be calculated. The first, $d^{\prime}$, is traditionally considered to measure discriminability (Green \& Swets, 1966), that is, the animal's ability to discriminate one class of stimuli from another. In the present situation, $d^{\prime}$ corresponds to the rat's ability to discriminate visited arms from those that have not yet been visited. The second, $\beta$, is a measure of bias (Green \& Swets, 1966), that is, the relative tendency to classify a stimulus in one manner rather than another because of factors other than the nature of the stimulus. In the present context, $\beta$ corresponds to the rat's tendency to classify arms as visited as opposed to unvisited independent of any information that is specific to a particular arm.

If a stricter criterion for choosing arms is adopted when the POI occurs later than when it occurs earlier, then a response to the manipulandum should be relatively more likely (and an arm visit should be relatively less likely) in the late POI conditions. If such a criterion shift exists, it would be reflected by a significant effect of POI on $\beta$. Likewise, if rats use only retrospective memory, as the criterion-shift hypothesis assumes, then the signal detection parameter $d^{\prime}$ should decrease as a function of POI. This is because the number of visits represented in working memory would increase as a function of POI, rendering it more difficult to discriminate visited arms from unvisited arms. The dual-code hypothesis, on the other hand, predicts a quadratic function relating POI and $d^{\prime}$. Discrimination of visited and unvisited arms would be 
relatively easy following early POIs (because a small amount of retrospective information would have been retained during the delay) and following late delays (because a small amount of prospective information would have been retained). The dual-coding hypothesis makes no prediction regarding an effect of POI on $\beta$.

In addition to trials on which the rats chose between an arm and the center manipulandum, the present experiment also included free-choice trials on which the rat was allowed to complete the maze following the delay. These trials served two functions. First, they might be necessary to maintain the contingencies of reinforcement that would produce a criterion shift or a change from retrospective to prospective coding (i.e, an increase in the probability of making an error as a function of choice number). Second, because the maze was rotated during the delay on half of these trials, they provide an opportunity to check for the use of intramaze cues (Olton \& Collison, 1979).

\section{METHOD}

\section{Subjects}

The subjects were 17 male Long-Evans hooded rats, approximately 6 months old at the beginning of the experiment. They were maintained at $80 \%-85 \%$ of their free-foeding weights by supplemental feeding immediately following each experimental session. A 12:12-h light:dark cycle was maintained in the colony. Experimental sessions occurred during the dark phase.

\section{Apparatus}

The apparatus was the same 12 -arm radial maze used by Cook et al. (1985). It consisted of $1280 \times 10 \mathrm{~cm}$ arms, radiating at equal angles from a central platform that was $41 \mathrm{~cm}$ in diameter. The maze was elevated $61 \mathrm{~cm}$ above the floor. A metal wall $(12.5 \mathrm{~cm}$ in height) surrounded the central platform. A circular hole $(7.5 \mathrm{~cm}$ in diameter) allowed access to each arm. Access to arms could be controlled individually by raising and lowering metal doors using a string-and-pulley assembly. A barrier $(12.5 \mathrm{~cm}$ in height and $26 \mathrm{~cm}$ in length) was mounted on one edge of each arm to prevent subjects from moving from arm to arm without returning to the center. Metal food cups (10 cm in diameter and $1.5 \mathrm{~cm}$ deep) were mounted at the end of each arm. During the testing phase of the experiment, a manipulandum constructed from one of the same food cups was located in the center of the maze. The food cup was mounted on top of small metal springs and a microswitch assembly. When pressed downward from any direction, this microswitch could produce delivery of two $45-\mathrm{mg}$ sucrose pellets (BioServe, Inc., product FOO42) into the cup from a pellet dispenser mounted approximately $12 \mathrm{~cm}$ above the center of the maze. The maze was located in a well-illuminated room with a variety of extramaze cues.

\section{Training Procedure}

The rats were first shaped to enter the arms of the maze. Food pellets were placed in the cups, on the arms, and in the center of the maze. Rats were placed in the maze in groups of 2-3 for $10 \mathrm{~min}$ per session. This shaping procedure was used for a minimum of two sessions.

Once a rat consistently ate pellets from the food cups, it was run individually in the standard radial maze task. Prior to each session, each arm was baited with two sucrose pellets. The doors to all 12 arms were opened, and the rat was allowed to visit arms until all 12 arms had been visited, 20 total choices had been made, or $10 \mathrm{~min}$ had elapsed. One session per day was run until the rat had completed the maze (visited all 12 arms) for 20 sessions. During this training phase, the center manipulandum was not mounted in the maze.

The rats were next trained to press the center manipulandum. A rat was placed in the center of the maze with the doors to all arms closed. Each response to the manipulandum produced two pellets. A session was terminated when 20 responses had been produced or $10 \mathrm{~min}$ had elapsed. Training continued until 20 responses occurred in five consecutive sessions.

\section{Testing Procedure}

Prior to each testing session, each arm was baited with two pellets. The rat was placed in the center of the maze with all doors closed and the center manipulandum present. Approximately $5 \mathrm{sec}$ after the rat was placed in the maze, the door to a single arm was opened. While the rat visited that arm, the door to a second arm was opened. Subsequently, the door to the next scheduled arm was opened and the door to the preceding arm was closed as the rat visited each arm. This procedure continued until the rats had visited $2,4,6$, 8 , or 10 arms. Following its last scheduled visit, the rat was removed from the maze and placed in a holding cage for $15 \mathrm{~min}$.

Following the delay interval, one of two procedures were used. In the single-arm procedure, the door to a single arm was opened. The rat was placed in the maze, directly between the open arm and the center manipulandum. If the open arm had not been visited prior to the delay, then it was baited with two food pellets and a response to the center manipulandum had no effect. If the open arm had been visited prior to the delay, then it was not baited and a response to the center manipulandum produced two food pellets. After making a response and consuming the food (if correct), the rat was removed from the maze for approximately $5 \mathrm{sec}$ while the door to the arm was lowered and the door to a second arm was raised. The rat was then placed back in the maze and allowed to make a choice between this second arm and the center manipulandum with the same contingencies as described above.

The second type of postdelay testing procedure was a free-choice procedure in which the doors to all 12 arms were opened. Those that had not been visited prior to the delay were baited. Center manipulandum responses had no effect. These trials were terminated following the 12 th correct choice or the 20 th choice. On half of these trials, the maze was rotated either $90^{\circ}, 180^{\circ}$, or $270^{\circ}$ during the delay. On these rotation trials, arms baited following the delay were those that were in the spatial locations that had not been visited prior to the delay.

Nine blocks of 12 sessions each were run. Within each block, 10 sessions included the single-arm testing procedure and 2 included the free-choice testing procedure. For the 10 single-arm sessions, each POI was in force for 2 sessions per block, and each response (arm visit vs. center manipulandum response) was correct an equal number of times. Within these constraints, the POI and the correct response were randomly determined. Likewise, the sequence of arms opened prior to the delay and the arms presented following the delay were randomly determined. For the 2 free-choice sessions, the POI was randomly chosen from among the five conditions used in the single-arm sessions. Within each block, the maze was rotated during one trial and not rotated during one trial (randomly chosen). The degree of rotation was randomly chosen from among the three possibilities.

\section{RESULTS}

\section{Training}

By the end of the initial 20 sessions of maze training, the rats were performing quite accurately. The mean num- 
ber of choices required to finish the maze during the last 5 sessions of training was 12.24 (range of individual means $=12-14$ ). An analysis was also carried out to determine the probability of erring as a function of choice number during these training trials. Figure 2 shows the results of this analysis. The mean probability of an error increased with choice number.

Center manipulandum training also proceeded smoothly. Rats met the training criterion during this phase in a mean of 8.2 sessions (range $=5-13$ ).

\section{Free-Choice Trials}

The mean number of choices taken to finish the maze in the free-choice trials when the maze was not rotated was 12.46. The mean number of choices to finish when the maze was rotated was 12.49 .

\section{Single-Arm Trials}

The data of primary interest come from the single-arm trials. Figure 3 shows the data in terms of the probability of an error as a function of POI. The probability of errors made when the maze arm was the correct choice (misses) and errors made when a center manipulandum response was the correct choice (false alarms) are shown separately. In addition, because another experiment using a somewhat similar procedure (Brown, 1987) found different patterns of results for the first and the second test of each session, the data are separated according to this variable as well. A POI $\times$ error type (miss vs. false alarm) $\times$ test (first vs. second) repeated measures analysis of variance (ANOVA) revealed an effect of POI $[F(4,64)=2.55, p<.05]$ and error type $[F(1,16)=8.64$, $p<.01]$, but no effect of test and no interactions. A trend analysis revealed that the effect of POI included a linear trend $[F(1,64)=4.70, p<.05]$, but not a quadratic trend.

To analyze the data in terms of $d^{\prime}$ and $\log \beta$, we calculated the proportion of hits (visiting baited arms) and the proportion of false alarms (visiting unbaited arms) for each rat separately for the first and second test. $d^{\prime}$ and $\log \beta$

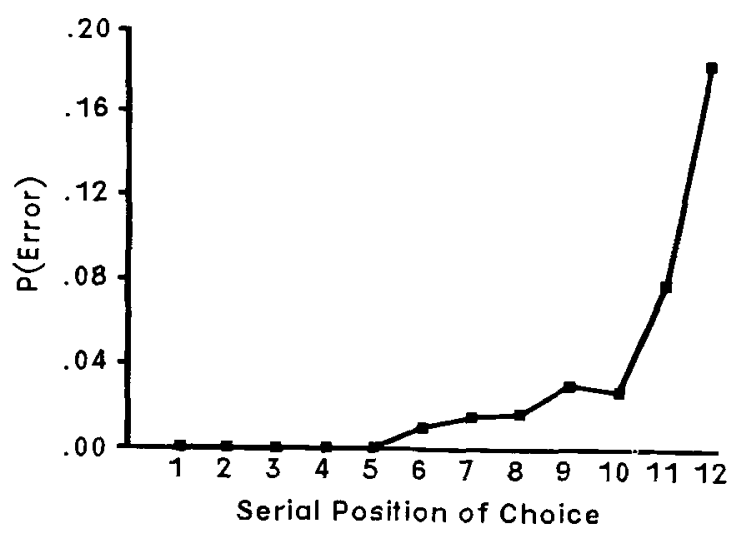

Figure 2. The mean probability of making an error as a function of choice number during training.

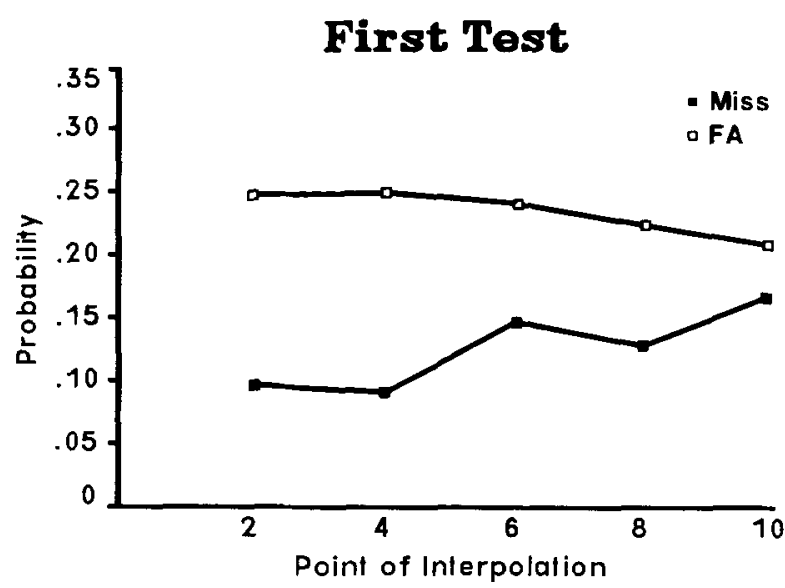

Second Test

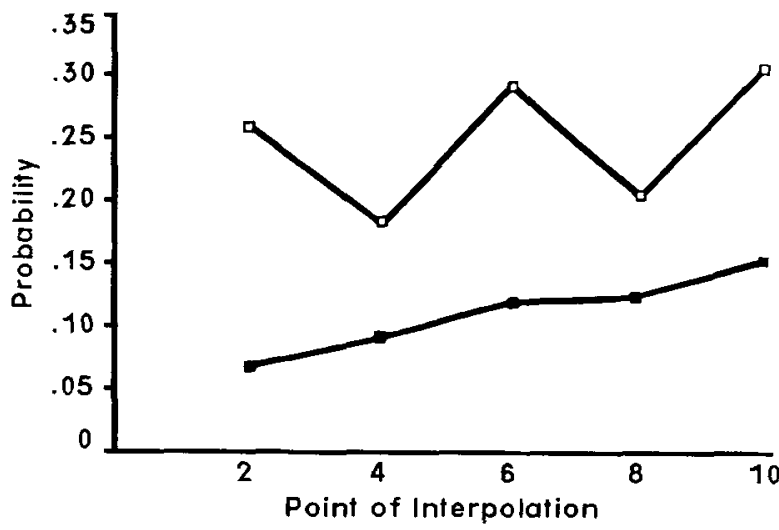

Figure 3. The mean probability of making an error in the singlearm tests as a function of point of delay interpolation. Data are shown separately for the first (top panel) and second (bottom panel) test of each session. $\mathbf{F A}=$ false alarm).

were then determined for both the first and second test at each POI for each rat. Use of the logarithm of $\beta$ is the accepted practice because of the asymmetrical range of values that $\beta$ can take (see McNicol, 1972, pp. 62-63). The mean values are shown in Figures 4 and 5.

An ANOVA performed on the $d^{\prime}$ data revealed a significant effect of POI $[F(4,64)=2.63, p<.05]$, but no effect of test (first vs. second) and no interaction. A trend analysis performed on the main effect of POI revealed a marginally significant linear trend $[F(1,64)=3.47, .10$ $>p>.05]$, but no quadratic trend.

An analogous ANOVA performed on the $\log \beta$ data revealed an effect of POI $[F(4,64)=3.26, p<.025]$, but no effect of test and no interaction. A trend analysis performed on the effect of POI revealed a significant linear trend $[F(1,64)=7.93, p<.01]$.

\section{DISCUSSION}

Despite the unique procedure of requiring rats to choose between a maze arm and a manipulandum response, they appeared to perform in a manner typical of rats in the 
radial maze. This is suggested by the uniformly high levels of accuracy in the free-choice sessions. In agreement with previous findings, there was no evidence of the use of intramaze cues in the present procedure, as shown by the lack of an accuracy difference in the rotation versus nonrotation free-choice trials (Olton \& Collison, 1979; Suzuki, Augerinos, \& Black, 1980; Zoladek \& Roberts, 1978). Thus, the present procedure did not appear to result in the use of processes that are radically different from those used in more standard radial maze tasks.

However, the results do differ from those of Cook et al. (1985) in that the function relating point of delay interpolation and postdelay choice accuracy was not quadratic in the present experiment. Instead, choice accuracy was lower for delays interpolated later in the choice sequence than for delays interpolated earlier in the choice sequence. This pattern of results is what would be expected if rats use only retrospective representations of maze arms during the delay. The discrepancy between the present results and those of Cook et al. can be explained by the criterionshift hypothesis. In the experimental procedures used by Cook et al., a stricter choice criterion in late POI conditions would result in an increase in correct rejections of previously visited arms at the microchoice level. Because more arms would tend to be evaluated, this would in turn increase the probability of considering, and correctly visiting, previously unvisited arms. In other words, following a late POI, the average number of microchoices that would occur between macrochoices would be greater, increasing the probability of a correct macrochoice. In the present procedure, on the other hand, only one arm is available. Therefore, any differences in the microchoice criterion should not affect choice accuracy (but see below).

If the preceding analysis is correct, then the effects of memory load and criterion should be revealed by measures of $d^{\prime}$ and $\beta$, respectively. Whereas memory load would affect the ability of the rat to discriminate a visited arm from an unvisited arm, the choice criterion would

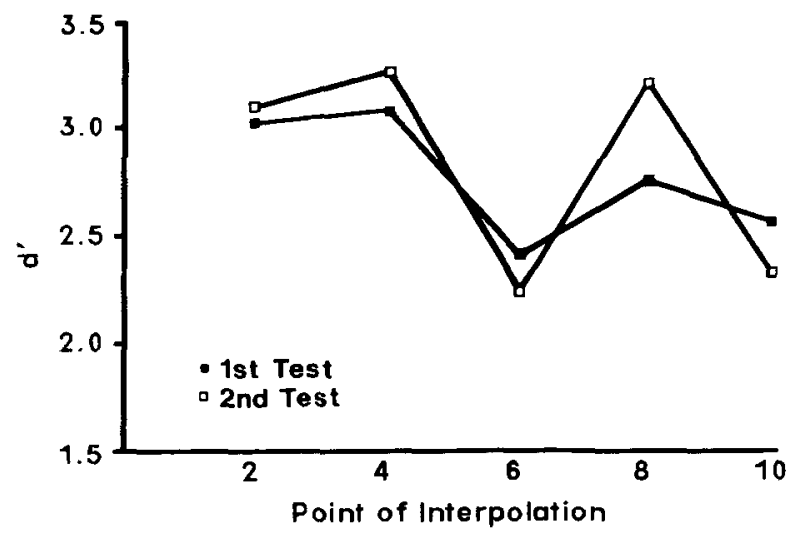

Figure 4. Performance measured in terms of $d^{\prime}$ during the singlearm tests as a function of point of delay interpolation. Data are shown separately for the first and second test of each session.

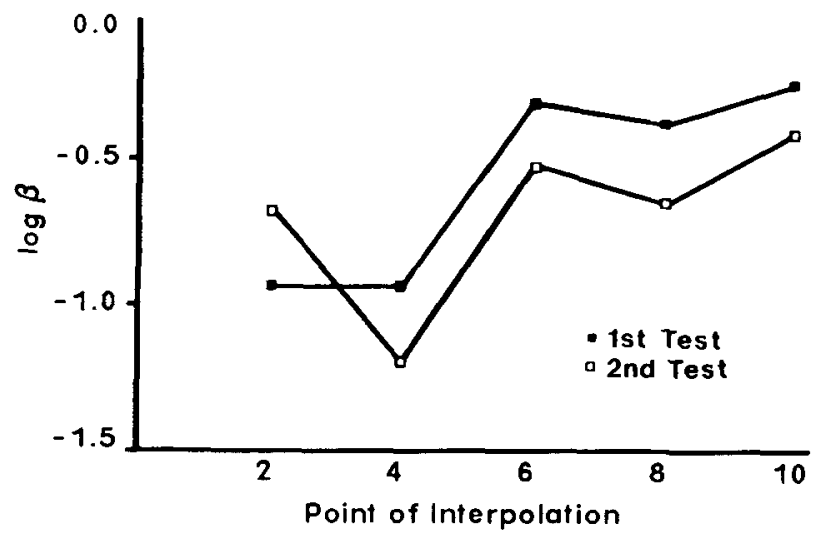

Figure 5. Performance measured in terms of the common $\log$ of $\beta$ during the single-arm tests as a function of point of delay interpolation. Data are shown separately for the first and second test of each session.

affect the tendency of the rat to visit the presented arm as opposed to pressing the center manipulandum, independently of that discriminatory ability. Thus, if the results of Cook et al. (1985) were produced by the dual-coding mechanism they described,then $d^{\prime}$ should be greater in early and late POI conditions than in the POI $=6$ condition. If the criterion-shift hypothesis is true, on the other hand, then $d^{\prime}$. should decrease with POI, and $\beta$ should indicate an increasing tendency to reject arms.

When analyzed according to these two indices, the present results provide no support for the dual-coding hypothesis. Although only a marginally significant trend was present in the $d^{\prime}$ data, the pattern of results suggests that the difficulty of discriminating previously visited arms from unvisited arms increases as a function of POI. These results are consistent with retrospective coding of maze arms, and contradict the earlier conclusion of Cook et al. (1985) that prospective coding is used as well. Thus, although the present data do not allow a firm conclusion regarding the relation between POI and $d^{\prime}$, the results are clearly different from those predicted by the dual-coding hypothesis. The results of the $\log \beta$ analysis, on the other hand, are quite clear. Rats visited the arm relatively more often following early delays and pressed the center manipulandum relatively more often following late delays. Thus, the tendency of the rats to visit an arm rather than press the manipulandum varied systematically as a function of POI. This finding is predicted by the criterionshift hypothesis.

As indicated in the introduction, consideration of how the probability of making an error in the standard radial maze task changes over the course of each trial provides a possible explanation for the development of the criterion shift. During the initial training phase, rats made more errors late in the choice sequence than early in the choice sequence (Figure 2). This may be due to psychological factors such as a buildup of proactive interference, but can also be attributed to the simple fact that the proportion of arms that remain baited decreases as a function 
of choice number. Previous analyses indicate that both of these factors play a role in choice accuracy (Olton \& Samuelson, 1976, Experiment 4). Whatever the explanation(s) for this phenomenon, it sets up contingencies that reinforce arm visits with a higher probability early in the choice sequence than late in the choice sequence. This difference in the probability of reinforcement as a function of choice number is the most likely explanation for the use of a criterion shift in the radial maze. Rats adopt a stricter criterion following late POIs than following early POIs because late choices were less likely to be reinforced during training.

The criterion-shift theory was originally proposed to explain the pattern of data described by Cook et al. (1985). They used two basic procedures for testing the ability of rats to choose correctly following the interpolated delay. First, in three experiments, the rat was allowed to choose freely from arnong all the maze arms until all 12 arms had been visited. Second, in a fourth experiment, the rat was given two-alternative forced choice (2AFC) tests in which only two arms (one previously visited and one unvisited) were available. Cook et al. argued that this $2 \mathrm{AFC}$ procedure provided the strongest evidence for the dual-coding hypothesis. Not only did the 2AFC procedure equalize the proportion of correct and incorrect arms available following the delay in the various POI conditions, but its use ruled out a criterion-shift explanation given the assumption that both "intervals" (arms) are fully examined prior to making a choice (Swets et al., 1961, p. 320). One of the reasons for conducting the present experiment was our concern that this assumption might not be valid. The present data reinforce this concern.

Although the criterion-shift hypothesis in its general form can be applied to either the free-choice or 2AFC procedure, choice behavior in the $2 \mathrm{AFC}$ procedure can be formally modeled in a much more straightforward manner. To explore the feasibility of the criterion-shift theory in its general form and to develop the theory in more detail, we present two such models that follow the logic used by Wright and Sands (1981) in their model of pigeon matching-to-sample performance.

\section{Model 1}

Using the nomenclature introduced in the introduction, the probability that the initial microchoice in the 2AFC procedure will result in a correct macrochoice is $.5\left(N_{n}\right)$, that is, the joint probability that the correct arm in the 2AFC test will be the object of the initial microchoice (.5) and that a correct arm will be visited when it is the object of a microchoice $\left(N_{n}\right)$. If it is assumed that each microchoice is independent of previous microchoices, then the probability of being correct on the second microchoice equals the probability of rejecting the arm considered during the first microchoice $\left[.5\left(O_{o}\right)+.5\left(N_{\circ}\right)\right]$ multiplied by the probability of a hit during the second microchoice, assuming that it occurs $\left(.5 N_{n}\right)$. In general, the probability of being correct on the $m$ th microchoice is

$$
.5 N_{n}\left(.5 O_{o}+.5 N_{o}\right)^{m-1} \text {. }
$$

The probability of being correct at the macrochoice level is simply the sum of the probabilities of being correct over microchoices, or

$$
\sum_{m=1}^{m=\infty}\left[.5 N_{n}\left(.5 O_{\circ}+.5 N_{o}\right)^{m-1}\right] .
$$

For example, assume that a previously unvisited arm will be accepted during a microchoice with a probability of $.9\left(N_{n}=.9\right)$ and that a previously visited arm will be rejected with a probability of $.7\left(O_{o}=.7\right)$. Equations 1 and 2 indicate that the probability of a correct macrochoice during the first microchoice is .45. A second microchoice will occur only if an arm is rejected during the first microchoice $\left(.5 O_{o}+.5 N_{o}=.40\right)$. The probability of a correct macrochoice as a result of a second microchoice is the joint probability that a second microchoice occurs (.40) and results in acceptance of the previously unvisited arm (.45), or .18. Extension of this example reveals that the probability of a correct macrochoice as a result of a 3rd, 4th, 5th, and 6th microchoice is .07, .03, .01, and $<.01$, respectively. As indicated by Equation 3, macrochoice accuracy is simply the sum of these probabilities (.74). As can be seen, as long as there is no strong bias to reject arms, the probability that a large number of microchoices will occur is very low. Thus, the predictions presented below are based on the first ten microchoices.

If the present single-arm test succeeded in producing the same probabilities of acceptance and rejection that occur in the microchoices made in the 2AFC procedure, and if the criterion-shift hypothesis is correct, then the data obtained in the present experiment should provide values for Equation 3 that will mimic the pattern of results obtained in Experiment 4 of Cook et al. (1985). $N_{n}$ is simply the observed percentage correct in the present experiment when the available arm had not been visited prior to the delay. Likewise, $O_{o}$ is the percentage correct when

Table 1

Prediction of Models in 2AFC Procedure with Microchoice Parameters Defined by Empirical Data

\begin{tabular}{cccccc}
\hline & \multicolumn{5}{c}{ POI Condition } \\
\cline { 2 - 6 } Test & 2 & 4 & 6 & 8 & 10 \\
\hline \multicolumn{6}{c}{ Model 1 } \\
1 & 78.3 & 78.4 & 78.0 & 79.3 & 79.7 \\
2 & 78.2 & 83.1 & 75.1 & 81.0 & 73.3 \\
\multicolumn{7}{c}{ Model 2 } \\
1 & 85.2 & 85.3 & 84.6 & 85.8 & 85.8 \\
2 & 85.5 & 89.2 & 82.1 & 87.2 & 80.1 \\
\hline
\end{tabular}

Note-Values shown are percentage correct. 
the available arm had been visited prior to the delay. The first two rows of Table 1 show the values obtained when the empirical data from the first and second tests of the present experiment are used in Equation 3. The differences between the predicted values for the various POI conditions are quite small. In the case of the data from the first test of each session, however, they do suggest an improvement in later POI conditions relative to the $\mathrm{POI}=6$ condition. This pattern is not obtained for the data from the second test of each session, however.

Perhaps the most likely explanation for the failure of Model 1 in conjunction with the present data to clearly predict the pattern of results found by Cook et al. (1985) is that the experimental procedure failed to fully isolate microchoices. Although rats had only one arm available, it is possible that multiple microchoices occurred in which the arm was evaluated repeatedly. Casual observation of the rats suggested that this was the case; rats often alternated between orientation toward the arm and behavior directed toward the manipulandum prior to making a choice. If multiple microchoices were in fact occurring even in the present single-arm procedure, this would have diluted the effect of the criterion shift by transforming it into an increase in accuracy in late POI conditions. Given this possible failure of the present procedure to fully isolate microchoices, it is reasonable to explore the ability of the model to predict performance in the $2 \mathrm{AFC}$ procedure when microchoice parameters are chosen to reflect a strong criterion shift at the microchoice level. We have done this and have found that the model can predict outcomes like those obtained by Cook et al. under a range of parameter values that conform to the criterion-shift theory. A sample of the model's predictions, given selected parameters, are shown in Figure 6.

\section{Model 2}

A slightly more complicated, but perhaps more realistic, model can be derived if one assumes that the same arm will not be the object of a microchoice twice in succession. In the $2 \mathrm{AFC}$ procedure, this means that a series of microchoices will involve alternating back and forth between the two available arms. Model 1 assumes that microchoices are independent, and hence does not make this assumption. The probability of being correct on the first microchoice, as in Model 1 , is $.5 N_{n}$. A rat can be correct on the second microchoice only if it considered the incorrect arm on the first microchoice and correctly rejected it; thus, the probability of being correct on the second microchoice is $.5\left(O_{0} \cdot N_{n}\right)$. Being correct on the third microchoice implies that there was an incorrect rejection of the correct arm on the first microchoice and a correct rejection of the incorrect arm on the second microchoice; thus, the probability of being correct on the third microchoice is $.5\left(N_{o} \cdot O_{o} \cdot N_{n}\right)$. This logic can be ex-
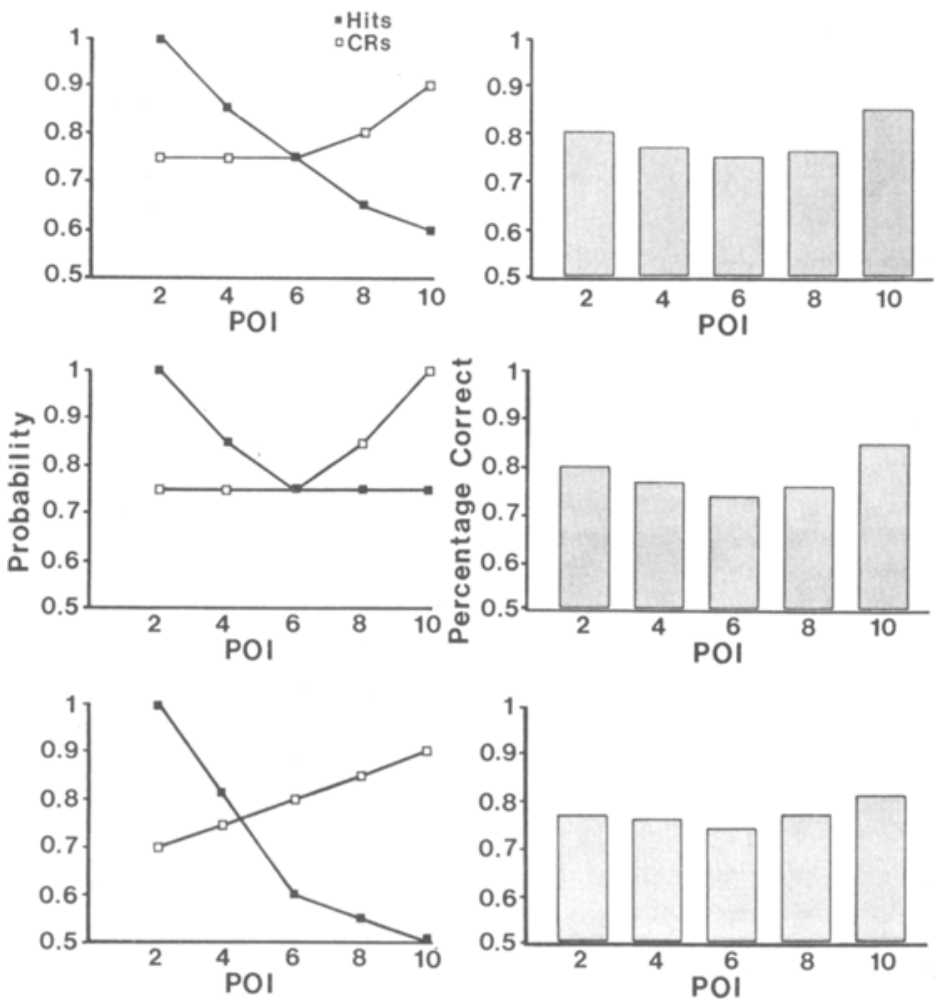

Figure 6. Predictions of Model 1 (right panels) given three sets of microchoice parameters (left panels). $\mathbf{C R}=$ correct rejection. 
trapolated to include additional microchoices and, as in Model 1, the probability of being correct at the macrochoice level is simply the sum of the probabilities over microchoices. The pattern of results in the 2AFC procedure predicted by this model, given the empirical data of the present experiment, is shown in the last two rows of Table 1. The predictions of this model are quite similar to those of Model 1, except that somewhat higher levels of accuracy are obtained.

\section{Conclusions}

Both the dual-coding theory proposed by Cook et al. (1985) and the criterion-shift theory proposed here have the potential of explaining the quadratic relation between accuracy and POI in the 12-arm radial maze. The $\log \beta$ analysis of the present experiment provides positive evidence that a criterion shift does occur in the radial maze, at least under the present conditions. The models developed above demonstrate that such a criterion shift could explain the 2AFC data of Cook et al., which, as they argue, is the strongest support for the dual-coding theory. On the other hand, the particular form of the criterionshift effect found in the present experiment does not emulate the previous data very well. In addition, the criterionshift hypothesis has no explanation for the serial position curves described by Cook et al. as converging evidence for the dual-coding hypothesis. Thus, the present work should be considered as preliminary, but as providing a viable alternative to the dual-coding hypothesis.

Aside from the value of the criterion-shift theory as a potential explanation of effect of point of delay interpolation, the fact that the tendency of rats to visit arms in the radial maze changes as a function of choice number underscores the complexity of the psychological processes involved in this widely used task. Although existing views of rat spatial ability focus on the role of memory, future theories may be forced to accommodate important influences of other factors.

\section{REFERENCES}

BowER, G. H. (1959). Choice point behavior. In R. R. Bush \& W K. Estes (Eds.), Studies in mathematical learning theory. Stanford, CA: Stanford University Press.

Brown, M. F. (1987). The effect of point of delay interpolation in a 16-arm radial maze. Unpublished manuscript.

Brown, M. F., \& Coox, R. G. (1986). Within-trial dynamics of radial maze performance in rats. Learning \& Motivation, 17, 190-205.
CoOK, R. G., Brown, M. F., \& Riley, D. A. (1985). Flexible memory processing by rats: Use of prospective and retrospective information in the radial maze. Journal of Experimental Psychology: Animal Behavior Processes, 11, 453-469.

GREEN, D. A., SWETS, J. A. (1966). Signal detection theory and psychophysics. New York: Wiley.

HoNIG, W.K. (1978). Studies of working memory in the pigeon. In S. H. Hulse, H. Fowler, \& W. K. Honig (Eds.), Cognitive processes in animal behavior (pp. 211-248). Hillsdale, NJ: Erlbaum.

Honig, W. K., Thompson, R. K. R. (1982). Retrospective and prospective processing in animal working memory. Psychology of Learning \& Motivation, 16, 239-283.

MCNicol, D. (1972). A primer of signal detection theory. London: Allen \& Unwin.

MUENZINGER, K. F. (1938). Vicarious trial and error at a point of choice: I. A general survey of its relation to learning efficiency. Journal of Genetic Psychology, 53, 75-86.

O'KeEFE, J., \& NADEL, L. (1978). The hippocampus as a cognitive map. Oxford: Oxford University Press.

OLton, D. S. (1978). Characteristics of spatial memory. In S. H. Hulse, H. Fowler, \& W. K. Honig (Eds.), Cognitive processes in animal behavior (pp. 341-373). Hillsdale, NJ: Erlbaum.

Olton, D. S., \& Colluson, C. (1979). Intramaze cues and "odor trails" fail to direct choice behavior on an elevated maze. Animal Learning \& Behavior, 7, 221-223.

Olton, D. S., SAmuelson, R. J. (1976). Remembrance of places past: Spatial memory in rats. Journal of Experimental Psychology: Animal Behavior Processes, 2, 97-116.

Riley, D. A., CooK, R. G., LAMB, M. R. (1981). A classification and analysis of short term retention codes in pigeons. Psychology of Learning \& Motivation, 15, 51-79.

Still, A. W. (1976). An evaluation of the use of Markov models to describe the behavior of rats at a choice point. Animal Behaviour, 24, 498-506.

Suzuki, S., Augerinos, G., \& Black, A. H. (1980). Stimulus control of spatial behavior on the eight-arm maze in rats. Learning \& Motivation, 11, 1-18.

Swets, J. A., Tanner, W. P., \& Birdsall, T .G. (1961). Decision processes in perception. Psychological Review, 68, 301-340.

Tolman, E. C. (1938). The determiners of behavior at a choice point. Psychological Review, 45, 1-41.

Wasserman, E. A. (1986). Prospection and retrospection as processes of animal short-term memory. In D. F. Kendrick, M. E. Rilling, \& M. R. Denny (Eds.), Theories of animal memory (pp. 53-75). Hillsdale, NJ: Erlbaum.

Wright, A. A., \& SANDS, S. F. (1981). A model of detection and decision processes during matching to sample by pigeons: Performance with 88 different wavelengths in delayed and simultaneous matching tasks. Journal of Experimental Psychology: Animal Behavior Processes, 7, 191-216.

ZOLADEK, L., RoBerTs, W. A. (1978). The sensory basis of spatial memory in the rat. Animal Learning \& Behavior, 6, 77-81.

(Manuscript received April 20, 1988; revision accepted for publication August 5, 1988.) 